\title{
A preformed basal lamina alters the metabolism and distribution of hyaluronan in epidermal keratinocyte "organotypic" cultures grown on collagen matrices
}

Accepted: 18 January 2000 / Published online: 7 March 2000

(C) Springer-Verlag 2000

\begin{abstract}
A rat epidermal keratinocyte (REK) line which exhibits histodifferentiation nearly identical to the native epidermis when cultured at an air-liquid interface was used to study the metabolism of hyaluronan, the major intercellular macromolecule present in basal and spinous cell layers. Two different support matrices were used: reconstituted collagen fibrils with and without a covering basal lamina previously deposited by canine kidney cells. REKs formed a stratified squamous, keratinized epithelium on both support matrices. Hyaluronan and its receptor, CD44, colocalized in the basal and spinous layers similar to their distribution in the native epidermis. Most (approximately 75\%) of the hyaluronan was retained in the epithelium when a basal lamina was present while most (approximately 80\%) diffused out of the epithelium in its absence. While REKs on the two matrices synthesized hyaluronan at essentially the same rate, catabolism of this macromolecule was much higher in the epithelium on the basal lamina (half-life approximately 1 day, similar to its half-life in native human epidermis). The formation of a true epidermal compartment in culture bounded by the cornified layer on the surface and the basal lamina subjacent to the basal cells provides a good model within which to study epidermal metabolism.
\end{abstract}

R.H. Tammi · M.I. Tammi · S. Pasonen

Department of Anatomy, University of Kuopio, 70211 Kuopio, Finland

V.C. Hascall · M. Hogg

Department of Biomedical Engineering, ND 20,

Cleveland Clinic Foundation, 9500 Euclid Avenue, Cleveland, Ohio 44195, USA

D.K. MacCallum (®)

Department of Anatomy and Cell Biology,

University of Michigan, Ann Arbor,

Michigan 48109-0616, USA

e-mail:dkmb@umich.edu

Tel.: +1-734-7635032, Fax: +1-734-7631166

\section{Introduction}

Hyaluronan (hyaluronic acid) is a macromolecule with a disaccharide repeat formed by the polymerization of alternating glucuronic acid and $\mathrm{N}$-acetylglucosamine residues. A single hyaluronan molecule can have a molecular weight of 10 million daltons (approximately 25,000 repeat disaccharides). Because of the large number of carboxyl groups on the glucuronic acid residues, hyaluronan functions as a large polyanion in the body with its open random coil structure occupying large solvent domains (Wight et al. 1991; Laurent et al. 1996). Hyaluronan is generally regarded as a component of connective tissue extracellular matrices and, indeed, in embryonic connective tissue, it can be the major structural molecule in the extracellular matrix where it plays roles in cell proliferation and migration (Toole 1991).

Recent studies have emphasized that hyaluronan is not simply a component of connective tissue extracellular matrices, but that it is also present in restricted locations within the nervous system (Ripellino et al. 1988; Bignami et al. 1993) and in certain epithelial tissues (Mani et al. 1992; Tammi et al. 1994a,b; Yung et al. 1996; Usui et al. 1999) including its universal presence within stratified epithelia (Tammi et al. 1988, 1990; Laurent et al. 1995; Wang et al. 1996; Hirvikoski et al. 1999). We (R.T. and M.T.) have studied the distribution of hyaluronan in the epidermis, a stratified squamous keratinizing epithelium. Hyaluronan is present in exceedingly high concentrations in the small intercellular spaces between adjacent basal and spinous keratinocytes, among the highest concentrations of any place in the body (Tammi et al. 1994b, 1999). Additionally, studies of human skin organ cultures have demonstrated that hyaluronan within the epidermis is rapidly turned over, an observation that suggests that the epidermis possesses mechanisms to catabolize hyaluronan that are closely coordinated with its synthesis by keratinocytes (Tammi et al. 1991).

In skin, both epidermal keratinocytes and the cells present in the dermis (principally fibroblasts) synthesize 
hyaluronan (Tammi et al. 1994b). Because both portions of the skin (epidermis and dermis) form and contain hyaluronan, it is frequently difficult to resolve the specific contribution of each component to the overall metabolism and content of this macromolecule in skin. To understand more completely the nature of hyaluronan synthesis and distribution in the epidermis, we have studied a rat epidermal cell line in culture. Our initial experiments utilized monolayer cultures to determine how hyaluronan is organized on the keratinocyte cell surface and its relationship to the cell surface hyaluronanbinding molecule, CD44 (Tammi et al. 1998).

We now extend these studies to the metabolism of hyaluronan in a reconstituted epidermis formed by the rat epidermal keratinocytes (REKs) when grown on collagen at the interface between the medium and gaseous phase of the culture (a "lifted" culture; Lillie et al. 1980). This culture conformation facilitates reformation of a stratified epithelium that more closely resembles the native epidermis than conventional "submerged" cultures and is often called an "organotypic" culture (Voigt and Fusenig 1979). Midway into our studies, it became apparent that while the REKs reformed an epidermis that closely resembled the native tissue, with basal cells dividing and moving through various cell strata ultimately to form a cornified ("keratinized") tissue surface, the epidermis remained "open" on its inferior aspect. In order to form a true epidermal compartment bounded superiorly by the stratum corneum and inferiorly by a basal lamina, we cultured the REKs on a collagen matrix that was covered by a basal lamina previously deposited by Maden Darby canine kidney (MDCK) cells which were then removed from the matrix prior to adding the keratinocytes. The presence of the basal lamina on the collagen matrix altered the metabolism and distribution of hyaluronan when compared to the "open" collagen matrix alone. A comparison of hyaluronan metabolism on the two different culture substrates and the barrier function of the basal lamina in altering the metabolism of hyaluronan are the subjects of this report.

\section{Materials and methods}

Keratinocyte culture

The REK cell line used in this study was derived from neonatal REKs (a gift of Howard Baden) originally isolated by Baden and Kubilus (1983). The keratinocytes stratify and terminally differentiate ("keratinize") in culture in the absence of "feeder" cells and exhibit a particularly high degree of tissue organization when cultured on collagen at the interface between the culture medium and culture gas phase (MacCallum and Lillie 1990). Stock cultures were grown in Dulbecco's modified minimal essential medium $(1 \mathrm{~g}$ glucose $/ \mathrm{l}), 10 \%$ fetal bovine serum, $50 \mu \mathrm{g} / \mathrm{ml}$ gentamicin sulfate ("complete medium") at $37^{\circ} \mathrm{C}$ in humidified $95 \%$ air $/ 5 \% \mathrm{CO}_{2}$. Keratinocytes were subcultured by incubating them for approximately $10 \mathrm{~min}$ at $37^{\circ} \mathrm{C}$ in $0.02 \%$ EDTA and $0.05 \%$ crystalline trypsin (Calbiochem, La Jolla, Calif., USA) in $\mathrm{Ca}^{2+}, \mathrm{Mg}^{2+}$-free Earle's balanced salt solution buffered to $\mathrm{pH} 7.4$ with $20 \mathrm{mM}$ HEPES.

\section{Collagen substrate}

Commercially available rat tail collagen (Sigma, St. Louis, Mo., USA) was dissolved at a concentration of $2.2 \mathrm{mg} / \mathrm{ml}$ in $0.034 \mathrm{~N}$ acetic acid at $4^{\circ} \mathrm{C}$ overnight. The dissolved collagen was dialyzed at $4^{\circ} \mathrm{C}$ against $2 \times 500$ volumes $0.2 \mathrm{M} \mathrm{NaCl}$ in $50 \mathrm{mM}$ TRIS- $\mathrm{HCl}$, $\mathrm{pH} 7.6$, over $24 \mathrm{~h}$. The dissolved collagen, $800 \mu \mathrm{l}$, was added to individual 24.5-mm-diameter tissue culture inserts (Costar Transwell; $3.0 \mu \mathrm{m}$ pore size). Collagen fibrils were formed by incubating the tissue culture inserts for $45 \mathrm{~min}$ at $37^{\circ} \mathrm{C}$ in a humidified atmosphere. The collagen gels thus formed were used directly without further treatment or, alternatively, were immersed in balanced salt solution at $4^{\circ} \mathrm{C}$ until needed.

\section{Preformed basal lamina}

MDCK cells were obtained from the American Type Culture Collection (Bethesda, Md., USA) and were subcultivated onto collagen gels in the tissue culture inserts $(200,000$ cells/24.5-mm insert). The MDCK cells were fed 3 times per week for 18-22 days using the same medium as that used for the keratinocytes. MDCK cells were removed using a protocol slightly modified from that of Huber and Weiss (1989) and Kramer et al. (1985). This required exposing the cells for approximately $10 \mathrm{~min}$ to a sterile $10 \mathrm{mM}$ TRIS-HCl hypotonic buffer that contained $0.1 \%$ bovine serum albumin (BSA) and $0.1 \mathrm{mM} \mathrm{CaCl}_{2}, \mathrm{pH}$ 7.5. The MDCK cells on the collagen were subsequently solubilized by incubating them in sterile $0.2 \%$ deoxycholate (Calbiochem) in the same buffer for $2 \times 5 \mathrm{~min}$ at $37^{\circ} \mathrm{C}$ followed by incubation in sterile $0.5 \%$ Nonidet $\mathrm{P}-40$ in the same buffer for $5 \mathrm{~min}$ at room temperature. After each incubation, the surface of the Transwell was gently washed using a Pasteur pipette and the detergent solution removed. When viewed with a phase microscope, there should be no morphological evidence of the MDCK cells after the second deoxycholate incubation. Following lysis of the cells, the Transwell culture surface was covered by a basal lamina, deposited by the MDCK cells, sitting on the surface of a collagen gel composed of reconstituted native collagen fibrils. The cleaned culture substrates are rinsed 3 times over $1 \mathrm{~h}$ in balanced salt solution followed by two changes of complete tissue culture medium over $0.5 \mathrm{~h}$ at $37^{\circ} \mathrm{C}$. Alternatively, the basal lamina-covered collagen gels can be stored in balanced salt solution at $4^{\circ} \mathrm{C}$ for several months and subsequently treated with complete culture medium immediately prior to use.

\section{"Lifted" or "organotypic" keratinocyte cultures}

Cultures of REKs just having reached confluence with little or no morphological evidence of stratification were subcultivated onto either collagen-covered (designated $\mathrm{BL}-$ ) or basal laminacoated/collagen-covered (designated BL+) Transwell culture inserts at a concentration of 150,000-200,000 REKs/24.5-mm insert. The subcultivated REKs were grown for 2 days with culture medium present both in the well beneath the Transwell insert as well as on the surface of the cells. The culture medium was subsequently removed from the surface of the cells and the level of the medium beneath the Transwell insert adjusted to the level of the REKs present on the BL- or BL+ collagen gels. The cultures for this study were grown for an additional 5 days at $37^{\circ} \mathrm{C}$ in humidified $95 \%$ air $/ 5 \% \mathrm{CO}_{2}$ with the medium being changed 3 times per week.

\section{Electron microscopy}

Culture substrates to be examined by scanning electron microscopy were fixed for $2 \mathrm{~h}$ at room temperature in $2.5 \%$ glutaraldehyde in $\mathrm{pH} 7.4,0.1 \mathrm{M}$ sodium cacodylate that contained $4 \%$ sucrose and $2 \mathrm{mM} \mathrm{CaCl}_{2}$. The substrates were then sequentially fixed in aqueous $1 \% \mathrm{OsO}_{4}, 1 \%$ tannic acid, $1 \% \mathrm{OsO}_{4}$ for $1 \mathrm{~h}$ each before being dehydrated through ascending concentrations of ethanol. The etha- 
nol was exchanged by three changes of hexamethyldisilazane from which the substrates were subsequently air dried. The substrates were mounted on stubs and sputter coated with platinum/palladium prior to scanning.

Cultures examined as either epoxy-embedded 1- $\mu$ m-thick light microscopic sections or as conventional electron microscopic thin sections were fixed for $2 \mathrm{~h}$ at $4^{\circ} \mathrm{C}$ in a 50:50 mixture of buffered $2.5 \%$ glutaraldehyde and $2 \%$ aqueous $\mathrm{OsO}_{4}$ with the final mixture containing $4 \%$ sucrose and $2 \mathrm{mM} \mathrm{CaCl}{ }_{2}$. The samples were dehydrated in ethanol and propylene oxide and embedded in epoxy resin. Sections were stained with either toluidine blue (light microscope) or uranyl acetate and lead citrate (electron microscope).

\section{Histochemical demonstration of hyaluronan}

A hyaluronan-specific probe, biotinylated hyaluronan binding complex (bHABC), was prepared from a 4-M guanidine- $\mathrm{HCl}$ extract of bovine articular cartilage after dialysis and digestion with trypsin, as described previously (Tammi et al. 1994b). The bHABC probe exhibited the biotinylated G1 domain of aggrecan and biotinylated link protein as the only silver stained and biotinylated proteins present when analyzed by SDS-PAGE.

Eight-millimeter circular biopsies were cut from individual culture inserts and fixed in $2 \%$ paraformaldehyde, $2.5 \%$ glutaraldehyde, $0.5 \%$ cetylpyridinium chloride, and $30 \mathrm{mM} \mathrm{NaCl}$ in $0.1 \mathrm{M}$ phosphate buffer, $\mathrm{pH} 7.4$, overnight. The biopsies were washed with buffer, dehydrated, and embedded in paraffin. Three-micronthick sections were cut, deparaffinized, and incubated in 1\% BSA in phosphate buffer for $30 \mathrm{~min}$. The sections were then incubated with bHABC $(3-5 \mu \mathrm{g} / \mathrm{ml}$ diluted in $1 \%$ BSA, $0.1 \mathrm{M}$ sodium phosphate, $\mathrm{pH} 7.4$ ) overnight at $4{ }^{\circ} \mathrm{C}$, washed with buffer 3 times (5 min each), and incubated with avidin-biotin-peroxidase (Vector Laboratories, Irvine, Calif., USA) for $1 \mathrm{~h}$ at room temperature. The sections were then incubated for $5 \mathrm{~min}$ in $0.05 \%$ 3,3'-diaminobenzidine $(\mathrm{DAB})$ and $0.03 \%$ hydrogen peroxide in the phosphate buffer. After washes, the sections were counterstained with Mayer's hematoxylin for $2 \mathrm{~min}$, washed, dehydrated, and mounted in DPX. The specificity of the staining was controlled by preincubating the sections with Streptomyces hyaluronidase (100 turbidity reducing units $/ \mathrm{ml}$ ) in $50 \mathrm{mM}$ sodium acetate buffer, $\mathrm{pH} 5.0$, containing protease inhibitors for $3 \mathrm{~h}$ at $37^{\circ} \mathrm{C}$ to remove hyaluronan from the tissue (Tammi et al. 1989). Other controls included sections reacted with the bHABC probe that was pretreated with hyaluronan oligosaccharides as described (Tammi et al. 1989) to block specifically the binding of the probe to endogenous hyaluronan in the sections.

\section{CD44 localization}

For the localization of CD44, the tissues were fixed in Histochoice (Amresco, Solon, Ohio, USA) for $2 \mathrm{~h}$, washed, dehydrated in graded ethanol, and embedded in paraffin. The sections were first treated for 5 min with $1 \% \mathrm{H}_{2} \mathrm{O}_{2}$ to block endogenous peroxidase, and then incubated with $1 \%$ BSA in phosphate-buffered saline (PBS) for $30 \mathrm{~min}$ to block non-specific binding. Thereafter the sections were incubated with monoclonal anti-CD44 (OX50; Biosource, Camarillo, Calif., USA; 1:50 dilution in 1\% BSA) overnight at $4{ }^{\circ} \mathrm{C}$, followed by sequential 1 -h incubations with biotinylated anti-mouse antibody (Vector; dilution 1:50), and avidinbiotin-peroxidase (Vector; 1:200 dilution). The color was developed with DAB as described above. The controls included sections treated in the same way but omitting the primary antibody, or by replacing it with non-immune mouse IgG.

\section{Metabolic labeling of REK cultures}

Five days after the REK cultures were configured at the gas-liquid interface (7 days after subcultivation onto the respective matrices), cultures were metabolically labeled with $20 \mu \mathrm{Ci} / \mathrm{ml}\left[{ }^{3} \mathrm{H}\right]$ glucos- amine and $100 \mu \mathrm{Ci} / \mathrm{ml}^{35} \mathrm{SO}_{4}$ (both compounds were from Amersham, Little Chalfont, UK) in complete tissue culture medium. Cultures were analyzed at $3,6,9,12,18$, and $24 \mathrm{~h}$ after labeling. In separate cultures, fresh medium without radiolabeled precursors was added at time 0 . At 15 or $24 \mathrm{~h}$, the radiolabeled precursors were then added in a small aliquot from a concentrated stock solution to give final concentrations equivalent to the continuously labeled cultures. In each case, these cultures were incubated for an additional $3 \mathrm{~h}$ giving labeling windows of $15-18 \mathrm{~h}$ and $24-27 \mathrm{~h}$. Addition of isotopes near the end of the experiment was done to ensure that any changes observed in metabolic labeling were not due to progressive dilution or exhaustion of the isotopes.

\section{Culture compartments defined}

Three "compartments" of the cultures (medium, epithelium, and matrix) were analyzed. As explained below, values for two of the compartments (medium and matrix) were combined and expressed as a single value. At the end of each labeling interval, the medium $(2 \mathrm{ml})$ was collected and the culture inserts were washed once with $0.5 \mathrm{ml}$ Dulbecco's PBS which was added to the medium (= medium compartment). Thereafter the cultures were immersed in $1 \mathrm{ml} 0.04 \%$ sodium EDTA in PBS for $5 \mathrm{~min}$ at $37^{\circ} \mathrm{C}$ to separate the epithelium from the matrix (Tammi and Tammi 1986). The epithelial sheets were mechanically lifted off the matrices (BL+ and $\mathrm{BL}-$ ) using a stereo microscope and fine needles to accomplish the separation. The epithelial sheets were analyzed separately (= epithelial compartment) and the EDTA separating solution was combined with the collagen or basal lamina + collagen matrix (= matrix compartment). All three compartments were analyzed for glycosaminoglycans. Some of the separated cultures were processed for light microscopy to ensure that the separation between the two compartments took place at the junction of the basal cells with the underlying matrix (data not shown). Data obtained from the "media" and "matrix" compartments have been combined and treated as a single value for each time point for the sake of simplicity in the presentation of graphs and to facilitate the comparison between the REKs cultured on the two different matrices (BL- or BL+).

Purification of the radiolabeled hyaluronan and glycosaminoglycans

Carrier hyaluronan (4 $\mu$ g in $40 \mu \mathrm{l}$, Healon; Pharmacia, Uppsala, Sweden) and 4 volumes $100 \%$ ethanol saturated with $\mathrm{NaCl}$ were added to each medium, matrix, and epithelial sheet. After $1 \mathrm{~h}$ at $-20^{\circ} \mathrm{C}$, precipitates were centrifuged for $15 \mathrm{~min}$ at $2500 \mathrm{~g}$. Each precipitate was suspended in $200 \mu \mathrm{l} 50 \mathrm{mM}$ sodium acetate, $\mathrm{pH} 5.8$, containing $5 \mathrm{mM}$ cysteine- $\mathrm{HCl}$ and $5 \mathrm{mM}$ sodium EDTA to which $50 \mu \mathrm{g}$ papain (Sigma) in $50 \mu \mathrm{l}$ water was added followed by incubation at $60^{\circ} \mathrm{C}$ for $4 \mathrm{~h}$. The samples were heated at $100^{\circ} \mathrm{C}$ for $10 \mathrm{~min}$, centrifuged at $13,000 \mathrm{~g}$ for $15 \mathrm{~min}$, and supernatants containing hyaluronan and other glycosaminoglycans recovered. Cetylpyridinium chloride ( $1 \%$ in $20 \mathrm{mM} \mathrm{NaCl}, 4$ volumes) was added to each supernatant followed by incubation for $1 \mathrm{~h}$ at room temperature. After centrifugation at $13,000 \mathrm{~g}$ for $15 \mathrm{~min}$, each supernatant was carefully removed by aspiration. Each precipitate was washed with $1 \mathrm{ml} \mathrm{H}_{2} \mathrm{O}$, centrifuged, and the supernatant discarded as above. Each cetylpyridinium chloride precipitate was dissolved in $50 \mu \mathrm{l} 4 \mathrm{M}$ guanidine- $\mathrm{HCl}$, and $900 \mu \mathrm{l}$ ethanol, saturated with $\mathrm{NaCl}$, was added. After $1 \mathrm{~h}$ at $-20^{\circ} \mathrm{C}$, each sample was centrifuged and the precipitate retained.

Each purified sample was dissolved in $50 \mu \mathrm{l} 50 \mathrm{mM}$ sodium acetate, $\mathrm{pH} 6.7$, and digested for $3 \mathrm{~h}$ at $37^{\circ} \mathrm{C}$ with $25 \mathrm{mU}$ chondroitinase $\mathrm{ABC}$ and $1 \mathrm{mU}$ of Streptococcal hyaluronidase (both from Seikagaku Kogyo, Tokyo, Japan), and 10-45 $\mu$ l injected onto a $1 \times 30 \mathrm{~cm}$ Superdex Peptide column (Pharmacia) and eluted at $0.5 \mathrm{ml} / \mathrm{min}$ with $0.1 \mathrm{M} \mathrm{NH}_{4} \mathrm{HCO}_{3}$. The eluent was monitored at $232 \mathrm{~nm}$, and aliquots of the $250-\mu \mathrm{l}$ fractions were counted for ${ }^{3} \mathrm{H}$ and ${ }^{35} \mathrm{~S}$. The carrier hyaluronan produced a disaccharide peak at 
$232 \mathrm{~nm}$ which was used to monitor the recovery (about $80 \%$ ) and correct for any losses in purification.

Ion-exchange chromatography

The radiolabeled disaccharide peaks from Superdex chromatography were dried, redissolved in water, and their identities confirmed by ion-exchange chromatography. The non-sulfated disaccharides and monosulfated chondroitin disaccharides were fractionated on $4 \times 50 \mathrm{~mm}$ Carbopak MA1 and PA1 columns (Dionex, Sunnyvale, Calif., USA) eluted isocratically with $20 \mathrm{mM}$ and $500 \mathrm{mM}$ sodium phosphate, $\mathrm{pH}$ 7.0, respectively. The disulfated disaccharides were chromatographed on a $0.4-1.0 \mathrm{M} \mathrm{LiCl}$ gradient on the PA1 column. More than $85 \%$ of the radioactivity in the dried peaks coeluted with standards (Seikagaku Kogyo) in the expected positions.

Chemical quantitation of hyaluronan with double labeling

The specific activity of $\left.{ }^{35} \mathrm{~S}\right]$ sulfate in the medium compartment is the same as that in $3^{\prime}$-phosphoadenosine- $5^{\prime}$-phosphosulfate, the metabolic precursor of sulfate incorporation into sulfated glycosaminoglycans, after a short equilibration time between the medi$\mathrm{um}$ and intracellular pools. Thus the ${ }^{35} \mathrm{~S}$ activity in chondroitin sulfate is a measure of the mass of chondroitin sulfate synthesized during the labeling period. Conversely, the specific activity of $\left[{ }^{3} \mathrm{H}\right]$ glucosamine in the medium is much higher, often more than 100 -fold, than the specific activity of the uridine diphosphate (UDP)- $N$-acetylhexosamine pool even after equilibration between the medium and intracellular compartments. This occurs because glucose is the primary source of glucosamine inside the cell, and therefore, metabolic conversion of glucose to glucosamine dilutes the specific activity of the exogenous, radiolabeled glucosamine. This dilution factor is generally unknown. However, we take advantage of the dual label in the chondroitin sulfate disaccharides to circumvent this problem.

From the known specific activity of the $\left.{ }^{35} \mathrm{~S}\right]$ sulfate in the medium, the masses of the monosulfated disaccharides were determined for each sample. Since the UDP- $N$-acetylhexosamine pool is a common precursor for both chondroitin sulfate and hyaluronan synthesis, the ratio of ${ }^{3} \mathrm{H}$ activity for these two glycosaminoglycans is equivalent to their mass ratio. Thus, the product of the mass of chondroitin sulfate synthesized (derived from the ${ }^{35} \mathrm{~S}$ activity) times the ratio of ${ }^{3} \mathrm{H}$ in hyaluronan to that in chondroitin sulfate is a measure of the mass of hyaluronan synthesized. The rationale and efficacy of this method is presented in greater detail in Yanagishita et al. (1989).

Determination of size distribution of hyaluronan using S1000 size-exclusion chromatography

Extracts of collagen were combined with the medium, and $0.5 \mathrm{ml}$ of this sample was used for size distribution determination. The samples were injected into a $1 \times 30 \mathrm{~cm}$ column of Sephacryl S1000 (Pharmacia), eluted with a flow rate of $20 \mathrm{ml} / \mathrm{h}$ with sodium acetate buffer $(0.15 \mathrm{M}, \mathrm{pH} 6.8)$ containing $0.1 \%$ CHAPS. Halfmilliliter fractions were collected and two samples of $100 \mu \mathrm{l}$ were taken for the assay of hyaluronan using CPC precipitation on nitrocellulose membrane as previously described (Ågren et al. 1997). One of the samples was subjected to digestion with Streptomyces hyaluronidase (5 turbidity reducing units/sample) for $3 \mathrm{~h}$ at $37^{\circ} \mathrm{C}$ before $\mathrm{CPC}$ precipitation, while the other sample got an equal volume of buffer. The difference in DPMs between the undigested and digested sample was used to represent counts in hyaluronan.

\section{Results}

\section{Culture substrates}

The basal lamina deposited on the collagen gel by MDCK cells exhibited a clean, smooth, featureless surface when examined at low power by scanning electron microscopy (Fig. 1A). When the surface of the collagen gel was gently scraped by fine forceps, the basal lamina was torn and the underlying collagen fibrils could be visualized (Fig. 1B,C). When the surface of the basal lamina was examined at high power faint, linear profiles that sometimes overlapped were observed (Fig. 1D,E). These profiles represented collagen fibrils immediately subjacent to the basal lamina that apparently were firmly adherent to the basal lamina because in no case were we able to peel just the basal lamina from the surface of the gel without accompanying collagen fibrils.

Occasional $\left(1-2 / 100 \mu \mathrm{m}^{2}\right)$, small $(<1.0 \mu \mathrm{m})$ circular or oval defects in the lamina were evident at high magnification (Fig. 1F). These defects apparently result from MDCK cell cytoplasmic processes projecting through the formed basal lamina (Valentich 1982). Thus, while a basal lamina covers the entire surface of the collagen gel, it is evident that the lamina is not $100 \%$ intact. [Our experience is that one of each batch of basal laminacovered collagen gels should be examined by scanning electron microscopy before using them as culture substrates because infrequent batches may exhibit many small holes (approximately 100/100 $\mu^{2}$ )]. The arrangement and space occupied by the collagen fibrils exposed by peeling back the basal lamina (Fig. 1C) was characteristic of the collagen matrix without a basal lamina used in these culture experiments, and was conspicuously "open" when compared to the basal lamina-covered collagen gels.

Morphology of REKs cultured on collagen or basal lamina-covered collagen at the air-medium interface (an "organotypic culture")

REKs subcultured onto either substrate grew to confluence, stratified, and exhibited the normal progression from basal cell to cornified surface cell (Fig. 2A,B). The number of cells in the viable cell strata remained relatively constant from 5 days of lifted culture onward; however, the cornified layer continued to increase in thickness as terminally differentiated cells were continually added for the duration of the culture period (not shown). The major morphological difference between REKs grown on collagen alone (BL-) and REKs grown on the basal lamina-covered collagen $(\mathrm{BL}+)$ was evident at the junction of the basal keratinocytes with the underlying matrix. Basal keratinocytes grown on collagen alone exhibited numerous, small cytoplasmic processes extending from the undersurface of the cell down into the meshwork of collagen fibrils. With extended culture, a sizable population of detached cytoplasmic fragments 

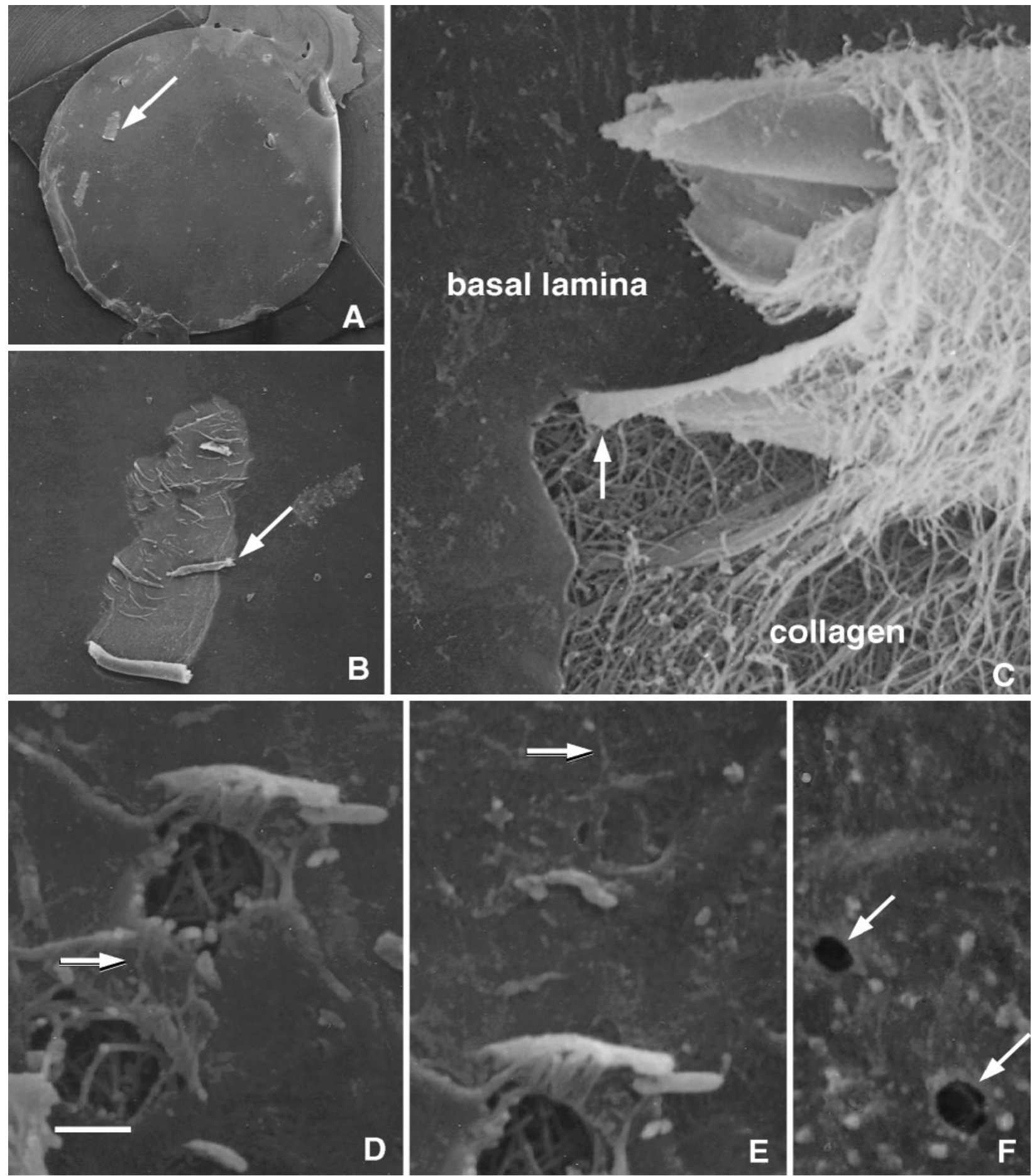

Fig. 1A-F Scanning electron micrographs of basal laminacovered collagen support matrices. A Eight-millimeter diameter sample of the basal lamina-covered matrix. Arrow points to the region shown in B. B Surface defect made by dragging fine forceps across the basal lamina exposing the underlying collagen. The edge indicated by the arrow is shown at higher power in $\mathbf{C}$. C Torn edge of the basal lamina (arrow). The solid surface of the basal lamina contrasts with the mesh work of collagen fibrils underlying it. Collagen fibrils always were torn away with the basal lamina when the surface was disturbed indicating that the basal lamina was firmly adherent to the collagen. The arrangement of the collagen fibrils in the micrograph is identical to that of matrices used to culture keratinocytes in the absence of a basal lamina (BL-). D,E High power scanning electron micrographs of the basal laminar surface indicating a faint fibrillar texture (examples at arrows) due to the underlying collagen fibrils. Each micrograph includes a surface defect made to expose underlying collagen fibrils. F Two pores (50 and $80 \mathrm{~nm}$; arrows) in the basal lamina due to processes of Maden Darby canine kidney cells extending through the basal lamina as it was being formed. Bar (in D) $1.7 \mathrm{~mm}$ in $\mathbf{A}$; $600 \mu \mathrm{m}$ in $\mathbf{B} ; 1.6 \mu \mathrm{m}$ in $\mathbf{C} ; 1 \mu \mathrm{m}$ in $\mathbf{D}-\mathbf{F}$ 


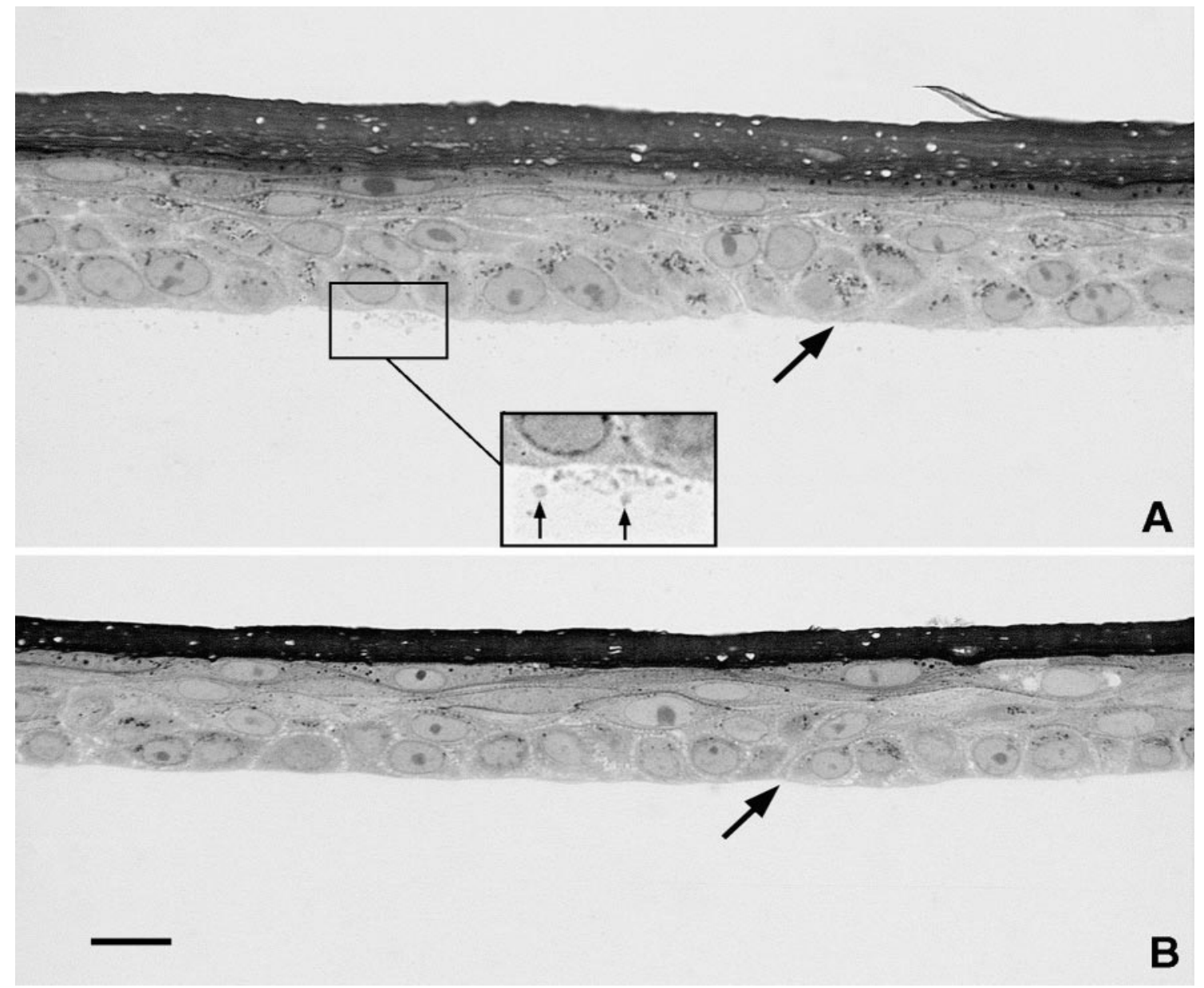

Fig. 2 Light micrographs of rat epidermal keratinocytes (REKs) cultured on collagen alone (BL-; $\mathbf{A})$ and on a basal lamina-covered collagen gel $(\mathrm{BL}+; \mathbf{B})$ after 5 days of growth at the air-liquid interface of the culture. Arrows mark the junction of the stratified epithelia with their support matrices. The upper part of the cornified layer was lost during processing of the BL+ culture $(\mathbf{B})$ and thus appears thinner than that on the BL-culture. Basal keratinocytes cultured on collagen (BL-) often released cytoplasmic fragments (arrows) from their undersurface into the collagen matrix (inset, A). Arrows mark the junction of the epithelium with the support matrix. Bar $15 \mu \mathrm{m}$

("blebs") accumulated immediately beneath the basal cells (Fig. 2A). Basal cells apposed to the basal lamina exhibited a smooth undersurface (Fig. 2B). Ultrastructural study of the REKs cultured on the two different substrata confirmed the light microscopic results. Basal cells on collagen alone exhibited cytoplasmic processes extending from the undersurface of the basal cells down among the collagen fibrils (Fig. 3A). Basal cells opposite the basal lamina appeared to have reestablished the normal basal keratinocyte relationship with the basal lamina and exhibited a smooth inferior plasma membrane surface with variable numbers of hemidesmosomes spaced along its length (Fig. 3B).
Hyaluronan and hyaluronan receptor (CD44) localization in organotypic cultures

Hyaluronan was identified in five 5-day organotypic cultures using a hyaluronan-specific bHABC purified from bovine articular cartilage. Hyaluronan was present between keratinocytes in either culture configuration $(\mathrm{BL}-, \mathrm{BL}+)$ in the spinous and basal cell layers with the strongest signal present between suprabasal spinous cells (Fig. 4A,B). Hyaluronan was demonstrable in the subjacent collagenous matrix either in a beaded pattern (not shown) or as a diffusely stained zone (Fig. 4A) in cultures grown on collagen alone (BL-), but was absent in cultures grown on a basal lamina-covered collagen matrix (BL+; Fig. 4B). The hyaluronan-binding molecule, CD44, localized immunohistochemically, exhibited a distribution in the cell layers nearly identical to that of hyaluronan in REKs cultured on either support matrix (Fig. 4C). CD44 was present in the BLcollagen matrix in the form of small globular deposits, presumably localized on the cytoplasmic blebs cast off from the basal keratinocytes (not shown).

Biosynthesis of glycosaminoglycans by REK organotypic cultures

The biosynthetic data presented are from a single experiment with one sample for each time point in each group 


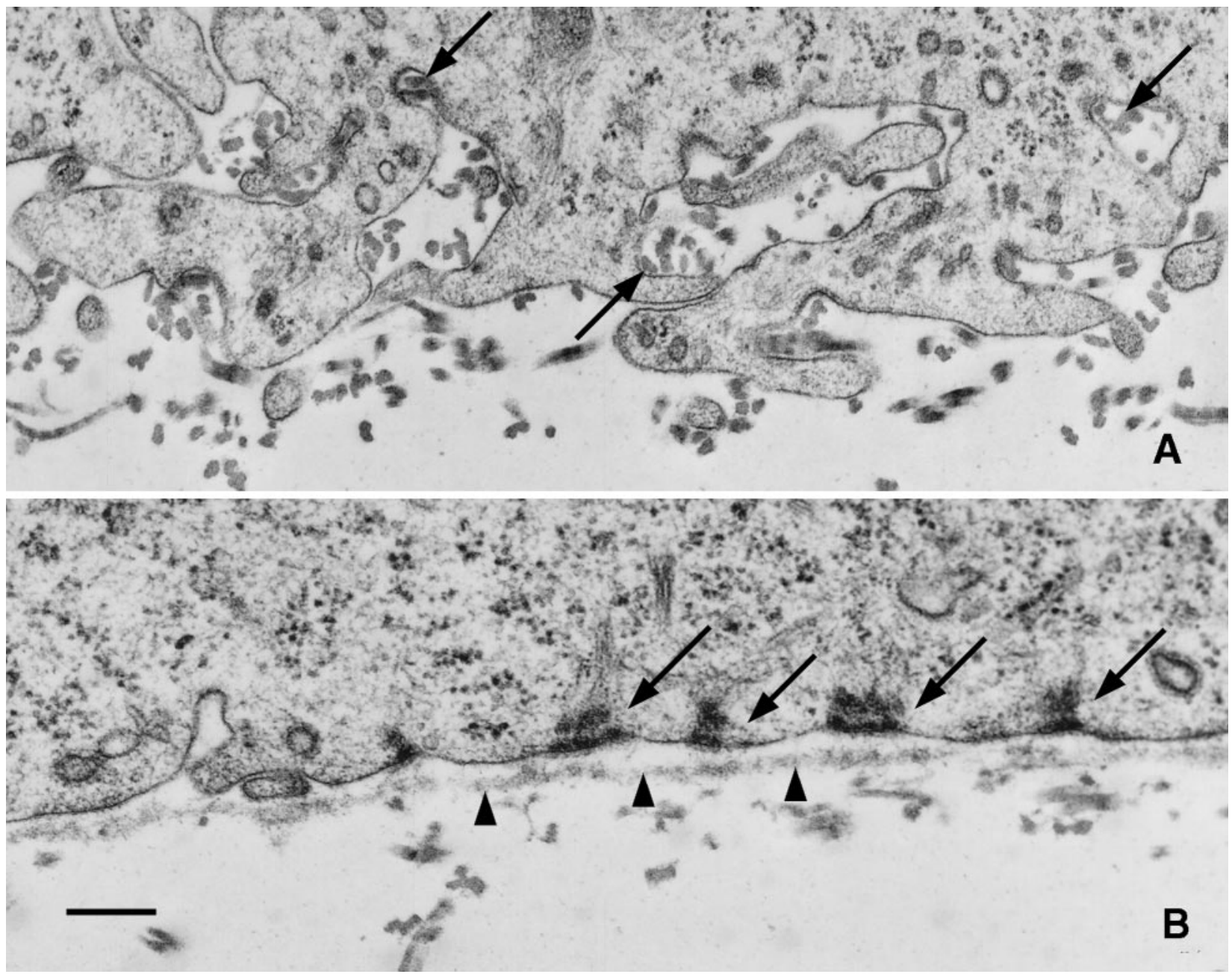

Fig. 3A,B The junction of basal keratinocytes with support matrices is illustrated. A Basal keratinocytes extend cell processes out into the underlying mesh of collagen fibrils, often enveloping groups of fibrils (arrows), when cultured on a collagen support matrix lacking a basal lamina. B The undersurface of a basal keratinocyte opposite a basal lamina (arrowheads) exhibits attachment specializations, hemidesmosomes (arrows), that are a normal feature of the native tissue. The "open" nature of the collagen support matrix (A; BL-) is evident when compared with boundary formed by the basal lamina (B; BL+). Bar $0.2 \mu \mathrm{m}$

$(\mathrm{BL}+, \mathrm{BL}-)$ together with cultures used in the labeling window experiments (16 total cultures, each of which yielded three components that were, in turn, then subjected to eight separate analyses). The data presented are consistent with two previous experiments that were conducted with fewer time points to establish labeling conditions and times.

Seven-day-old organotypic cultures of REKs were incubated for different times with ${ }^{[35}$ S $]$ sulfate and $\left[{ }^{3} \mathrm{H}\right]$ glucosamine as metabolic precursors for labeling glycosaminoglycans. The total incorporation of each precursor into purified glycosaminoglycans is shown in Fig. 5 as a function of labeling time and for individual 3 -h labeling windows $(0-3,12-15,24-27 \mathrm{~h})$. Incorporation of ${ }^{35} \mathrm{~S}$ into sulfated glycosaminoglycans (chondroit- in sulfate and heparan sulfate) decreased with labeling time, but showed no difference between cultures with $(\mathrm{BL}+)$ or without (BL-) an intervening basal lamina. While incorporation of ${ }^{3} \mathrm{H}$ activity into glycosaminoglycans also decreased with labeling time, there was a distinct difference between the two culture configurations with significantly less label in the BL+ configuration at times greater than approximately $10 \mathrm{~h}$.

The differences in ${ }^{3} \mathrm{H}$ incorporation into the glycosaminoglycans at the 12- to 24-h time points do not reflect different incorporation rates during that time period of the experiment because ${ }^{3} \mathrm{H}$ incorporation at each of the 3-h labeling windows was the same for both culture configurations. However, there was a gradual decrease in net incorporation from the first labeling window $(0-3 \mathrm{~h})$ to the final labeling window (24-27 h) in both culture configurations, most likely reflecting decreases in medium components, for example, glucose, necessary for glycosaminoglycan synthesis.

\section{Quantitation of glycosaminoglycans}

Portions of the purified glycosaminoglycans from each compartment (epithelium, matrix + medium) of each cul- 

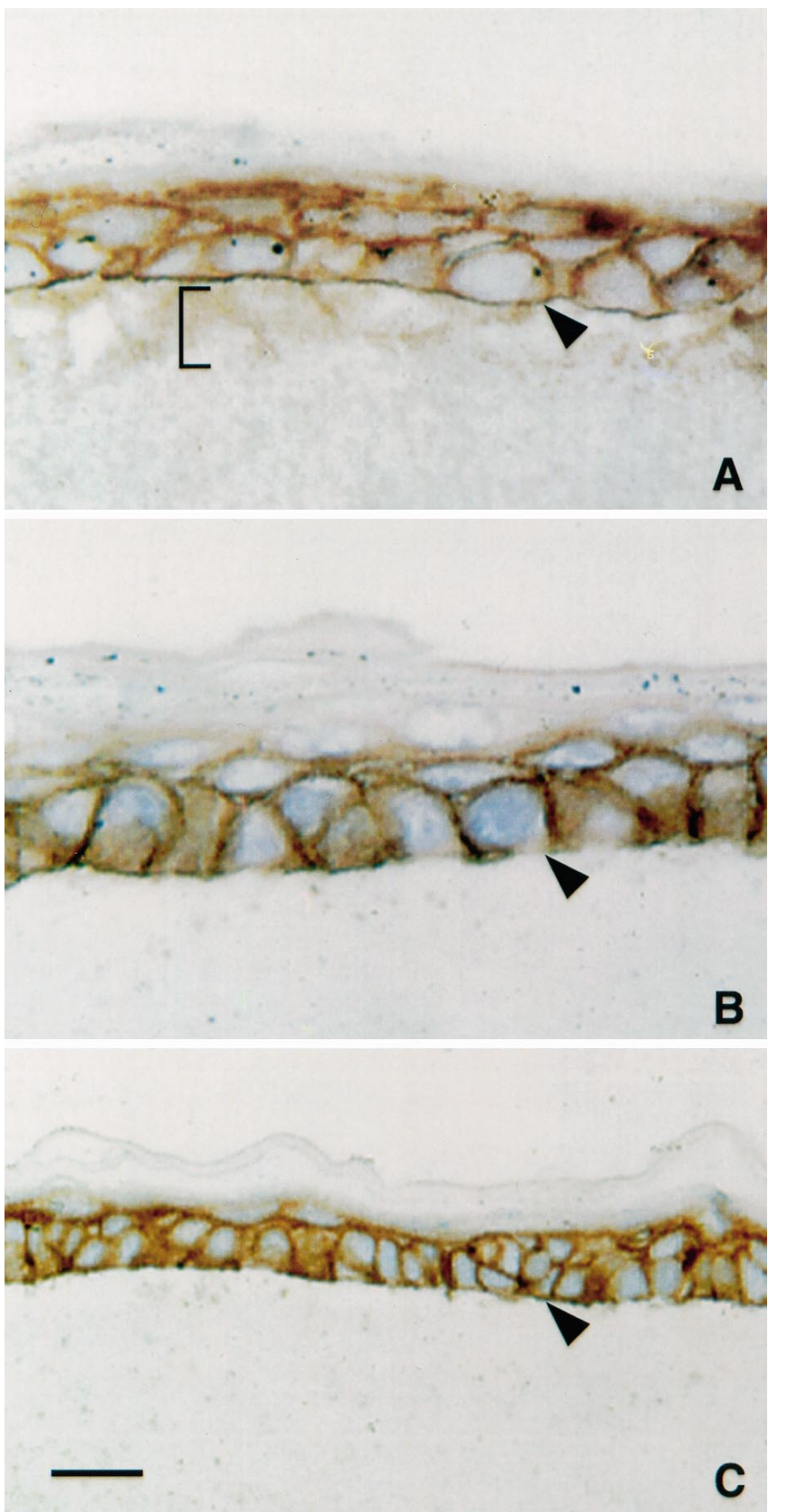

Fig. 4 Hyaluronan in REK cultures lacking a basal lamina $(\mathrm{BL}-; \mathbf{A})$ and with a basal lamina $(\mathrm{BL}+; \mathbf{B})$. In cultures lacking a basal lamina hyaluronan diffused into the collagen matrix immediately subjacent to the epithelium (bracket) while no hyaluronan was observed in this location in the BL+ cultures. Panel $\mathbf{C}$ demonstrates the presence of the hyaluronan-binding molecule CD44 in the same regions in which hyaluronan is present in both culture configurations. Arrowheads mark the junction of the epithelium with the support matrix. Bars $14 \mu \mathrm{m}$ in $\mathbf{A}, \mathbf{B} ; 20 \mu \mathrm{m}$ in $\mathbf{C}$

ture configuration (BL-, BL+) were digested with a combination of chondroitinase $\mathrm{ABC}$ and Streptococcal hyaluronidase. Each digest was eluted on a Superdex Peptide gel filtration column, an example of which is shown in Fig. 6. The three classes of glycosaminoglycans resolve as indicated, with intact heparan sulfate
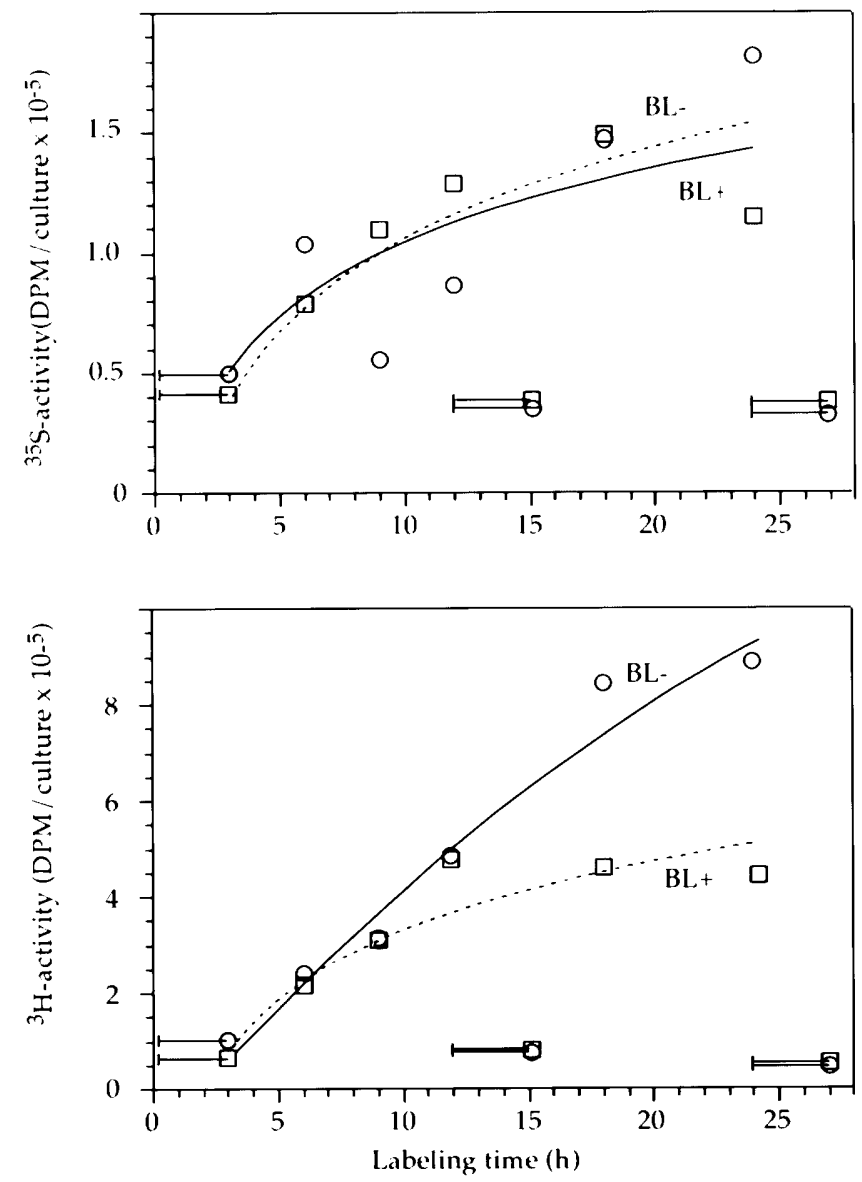

Fig. 5 Incorporation of radioactive ${ }^{35} \mathrm{SO}_{4}$ (upper graph) and ${ }^{3} \mathrm{H}\left(\left[{ }^{3} \mathrm{H}\right]\right.$ glucosamine as precursor; lower graph) as precursors into glycosaminoglycans synthesized in organotypic cultures of epidermal keratinocytes on collagen gels with $(\mathrm{BL}+=$ squares $)$ or without $(\mathrm{BL}-=$ circles $)$ an intervening basal lamina. The curves show logarithmic fitting of the data points (dotted line $=\mathrm{BL}+$; solid line $=\mathrm{BL}-$ ) for the 3- to 24-h labeling periods. Horizontal bars indicate values obtained from separate 3 -h labeling windows $0-3$, 12-15, and 24-27 $\mathrm{h}$ after starting the continuous labeling

chains eluting in the void volume and the disaccharides derived from chondroitin sulfate eluting as two separate peaks immediately before the disaccharides derived from hyaluronan. The hyaluronan disaccharide peaks represented a high proportion of the total ${ }^{3} \mathrm{H}$ activity (55-70\%) indicating that hyaluronan is the predominant glycosaminoglycan synthesized by the REKs.

The ratio of ${ }^{3} \mathrm{H}$ to the mass of monosulfated disaccharide (derived from ${ }^{35} \mathrm{~S}$ activity) is a measure of the specific activity of the galactosamine incorporated into chondroitin sulfate during the labeling period as described in Materials and Methods (Yanagishita et al. 1989). Values for this ratio are shown in Fig. 7 for the labeling experiment. For both culture configurations the values gradually increase approximately twofold during the $24 \mathrm{~h}$ of the experiment reflecting the slower equilibration of $\left[{ }^{3} \mathrm{H}\right]$ glucosamine than of $\left[{ }^{35} \mathrm{~S}\right]$ sulfate between the medium and intracellular pools. Importantly, however, there is no significant difference in this parameter be- 


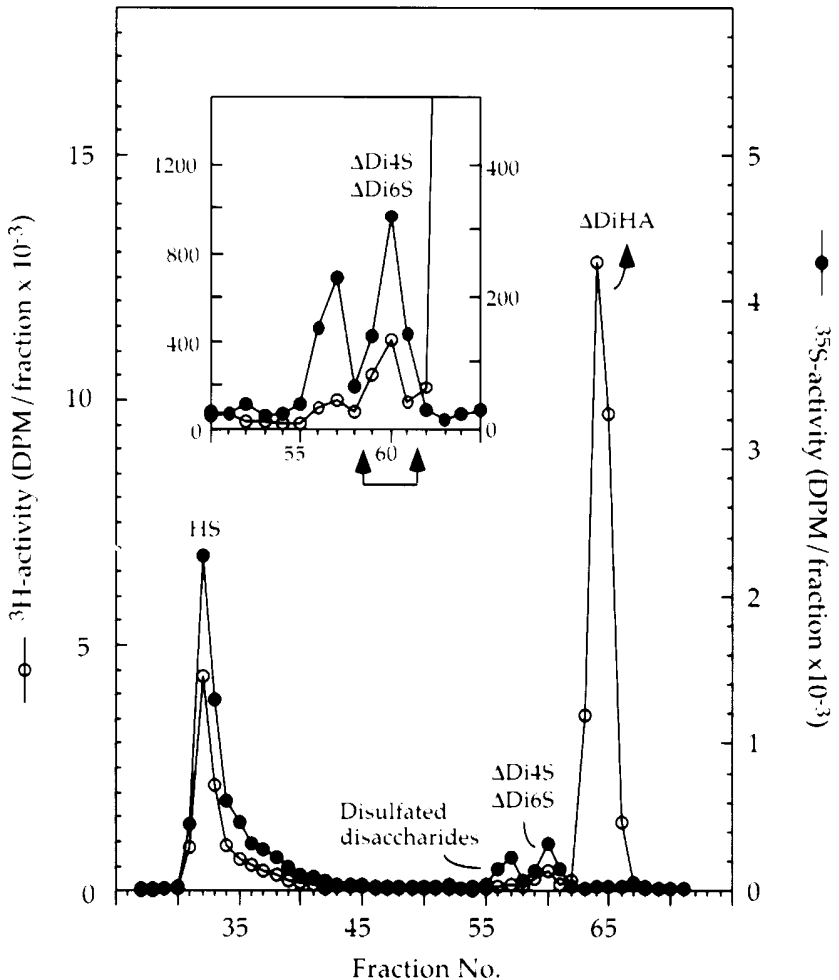

Fig. 6 An example of a chromatographic analysis of glycosaminoglycans synthesized by keratinocytes in an organotypic culture. Purified ${ }^{35} \mathrm{~S}$ - and ${ }^{3} \mathrm{H}$-labeled glycosaminoglycans were digested with chondroitinase $\mathrm{ABC}$ and Streptococcal hyaluronidase and separated on a Superdex Peptide gel filtration column. Undigested material, mainly heparan sulfate $(H S)$, appears in the void volume and disaccharides elute in the order of decreasing sulfation, as indicated in the figure. The ${ }^{35} \mathrm{~S}$ radioactivity in the monosulfated chondroitin sulfate disaccharides $(\triangle \mathrm{Di} 4 \mathrm{~S}$ and $\triangle \mathrm{Di} 6 \mathrm{~S}$, fractions 59-61, see inset) were used to calculate the specific activity of the incorporated ${ }^{3} \mathrm{H}$ and the chemical quantity of hyaluronan in the non-sulfated disaccharide peak $(\Delta \mathrm{DiHA})$

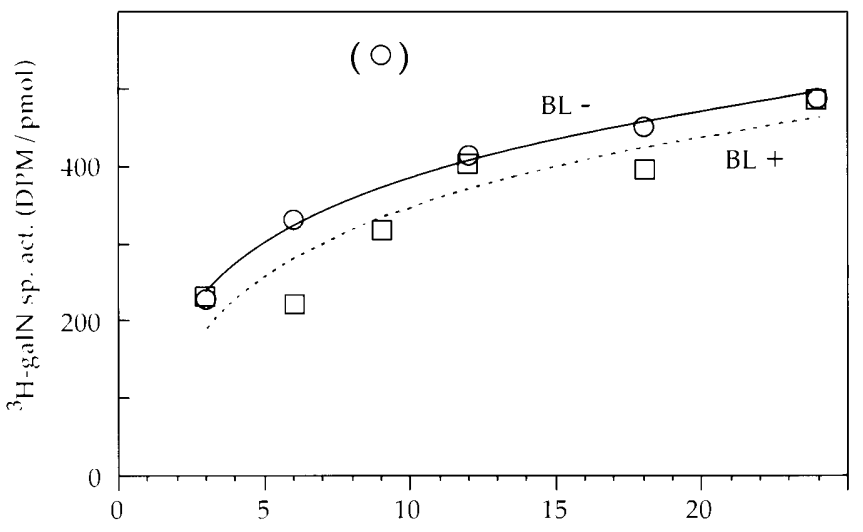

Fig. 7 Specific activity of $N$-acetylgalactosamine incorporated into chondroitin sulfate in organotypic cultures of epidermal keratinocytes. The values were obtained from the epithelium of cultures labeled for 3-24 h, each chromatographed as demonstrated in Fig. 6. The curves represent logarithmic fitting of the data points (excluding the value in parenthesis). For symbols and labels, see Fig. 5
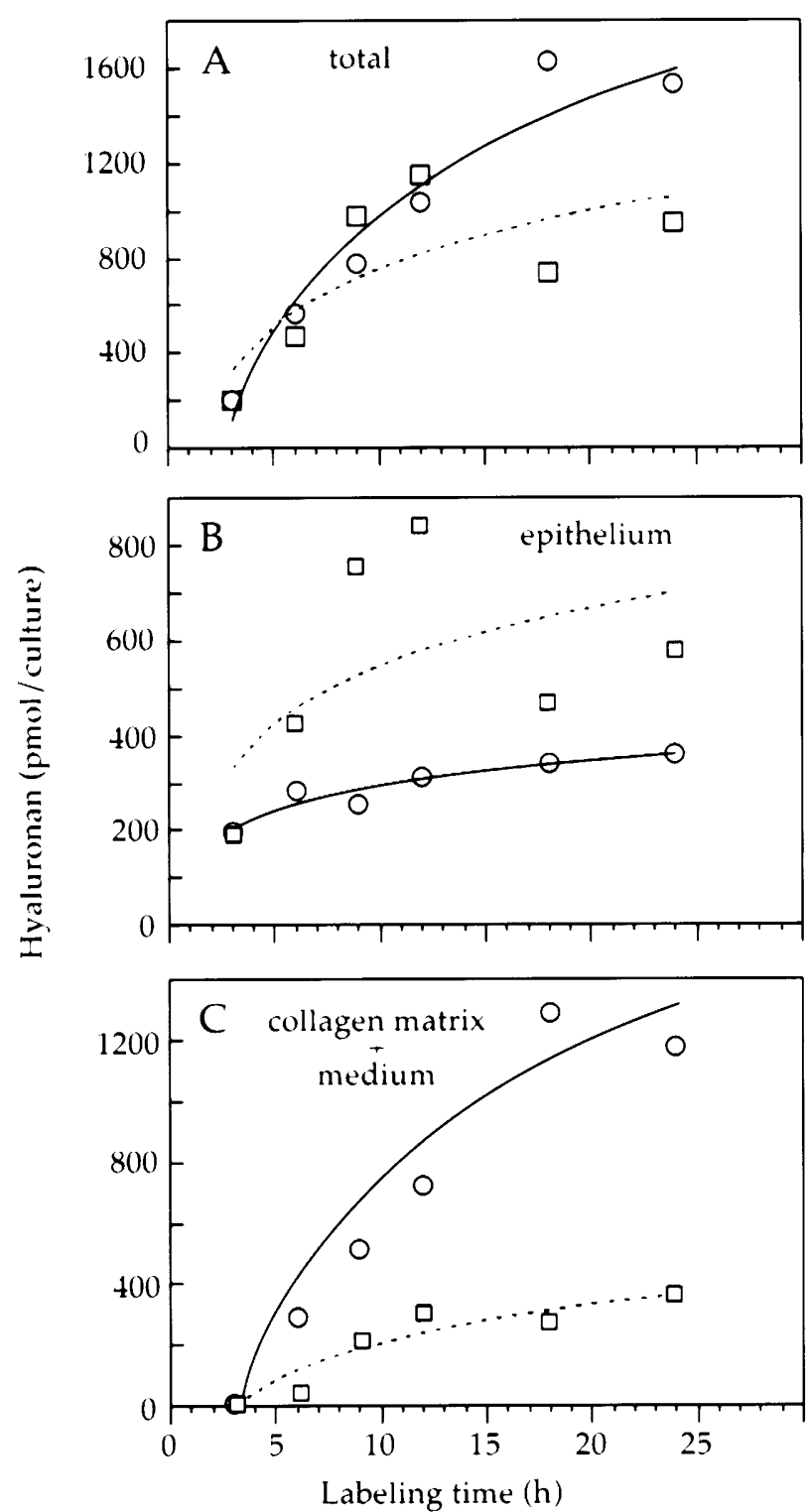

Fig. 8 Newly synthesized hyaluronan (total; A), and amounts in the epithelium (B) and the subjacent matrix plus medium compartments of organotypic keratinocyte cultures (C). The curves show the data points fitted to logarithmic equations. For symbols and labels, see Fig. 5

tween the two culture configurations (BL-, BL+). This means that differences in the distribution of ${ }^{3} \mathrm{H}$-labeled hyaluronan in the two different culture configurations reflect real differences in the amounts of the ${ }^{3} \mathrm{H}$-labeled hyaluronan and are not due to differences in specific activities of the precursor pools.

\section{Compartmentalization of hyaluronan}

Figure $8 \mathrm{~A}$ shows that the total ${ }^{3} \mathrm{H}$ activity, hence the total mass, of hyaluronan that accumulates over $24 \mathrm{~h}$ is approximately $35 \%$ greater in REK cultures without a basal lamina (BL-) than in cultures with a basal lamina (BL+). 

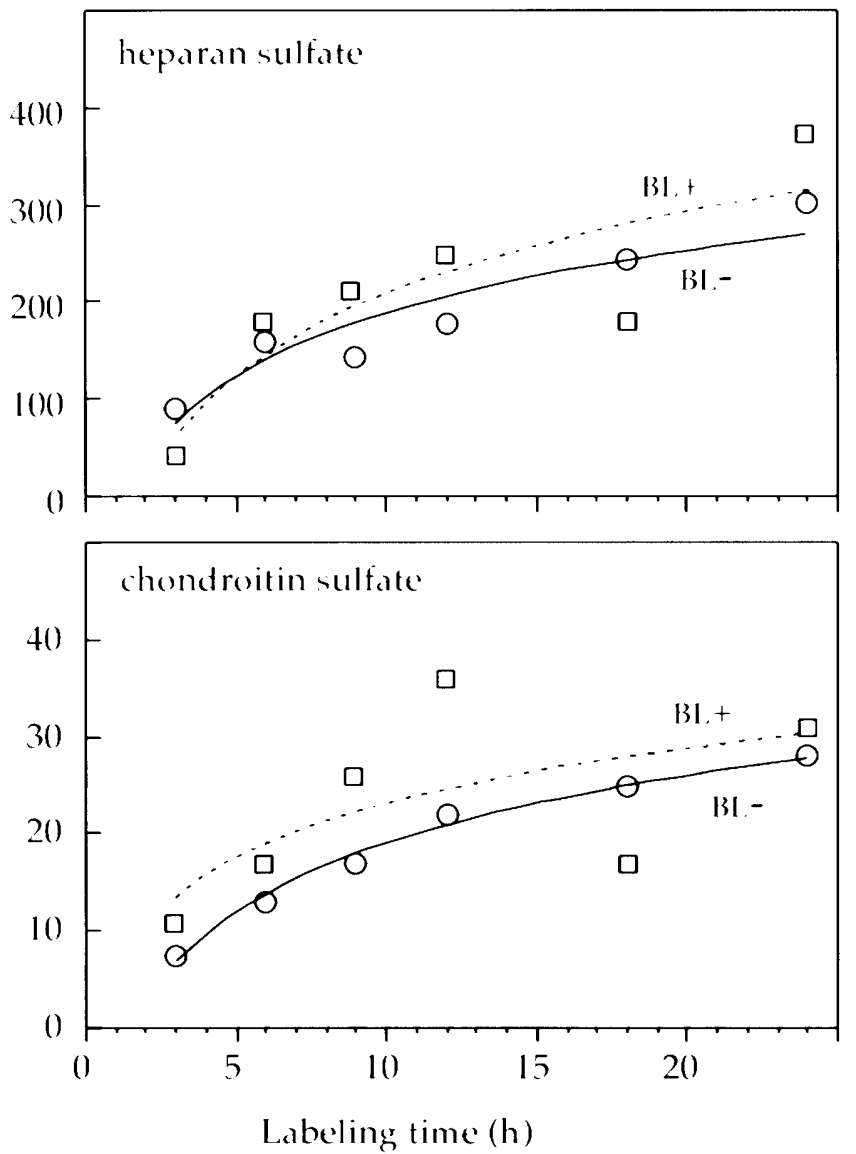

Fig. 9 Newly synthesized heparan sulfate (upper panel) and chondroitin sulfate (lower panel) in the epithelium of organotypic keratinocyte cultures. The values were based on separation of the glycosaminoglycans as shown in Fig. 6, and the specific activities of incorporated ${ }^{3} \mathrm{H}$ in Fig. 7. For symbols and labels, see Fig. 5

Inspection of the earliest time points (up to $6 \mathrm{~h}$; Fig. 8A) as well as the data in Fig. 5 indicate that there is no significant difference between the rates of hyaluronan synthesis between the two culture configurations. Therefore the results in Fig. 8A indicate that cultures with a basal lamina are catabolizing significantly more of the hyaluronan that was synthesized during the labeling period. Importantly, the amount of hyaluronan that is retained in the epithelial compartment of cultures with a basal lamina at $24 \mathrm{~h}$ is much higher (Fig. 8B). In this case approximately $75 \%$ of the total labeled hyaluronan is in the epithelial compartment. Conversely, only approximately $20 \%$ of the total hyaluronan is retained in this compartment in the cultures lacking a basal lamina; most has diffused into the underlying collagen matrix and medium compartments (Fig. 8C).

Heparan sulfate and chondroitin sulfate in the epidermal compartment

In contrast to hyaluronan, there were no differences in the accumulation of labeled heparan sulfate and chon-

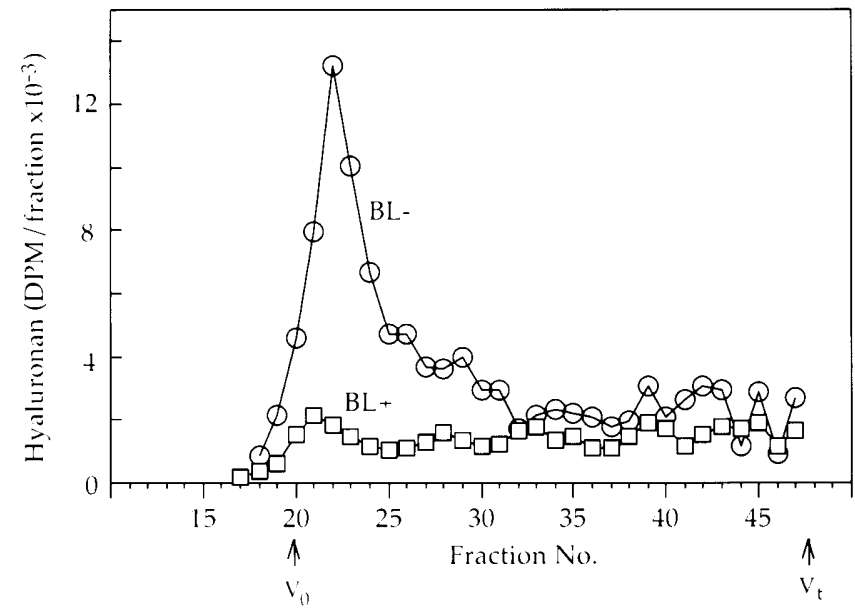

Fig. 10 Size distribution of hyaluronan in the matrix plus medium compartments of epidermal keratinocyte organotypic cultures. Proportional aliquots of extracts of the collagen gel and medium were combined and injected onto a $1 \times 30-\mathrm{cm}$ Sephacryl S1000 column eluted with $150 \mathrm{mM}$ sodium acetate, 0.1\% CHAPS, pH 7.0. Each fraction was analyzed for hyaluronan, based on its susceptibility to Streptomyces hyaluronidase. Arrows indicate the void $\left(V_{0}\right)$ and total volume $\left(V_{t}\right)$ of the column. For symbols and labels, see Fig. 5

droitin sulfate within the epidermal compartment of the two culture configurations (Fig. 9). In each culture condition, the accumulation continued, but with a gradually decreasing rate. The curves suggest that some of these glycosaminoglycans are also being catabolized, but additional experiments would be required to confirm this and to define approximate half-lives. The results also indicate that the keratinocytes synthesize approximately tenfold more heparan sulfate than chondroitin sulfate.

\section{Barrier function of the basal lamina}

Portions of extracts of the collagen matrix compartment were combined with equivalent portions of medium for cultures from the 24-h time points. Aliquots were then eluted from a Sephacryl S1000 column and fractions analyzed for content of hyaluronan. Figure 10 shows a major peak eluting near the void volume of the column for the sample from the cultures without a basal lamina (BL-), indicating the presence of high molecular weight hyaluronan. In contrast, very little high molecular weight hyaluronan was observed in the sample from the cultures with a basal lamina (BL+) although high molecular weight hyaluronan was present in the epithelium (data not shown). This demonstrates that the basal lamina deposited by the MDCK cells formed an effective barrier to high molecular weight hyaluronan.

\section{Discussion}

Growing epidermal keratinocytes on a support matrix at the interface between the gaseous and liquid phases of a 
culture greatly facilitates reformation of a tissue that closely resembles an epidermis. There are many variations used in such "organotypic" cultures with respect to the supporting matrices employed and the inclusion of viable connective tissue cells (see Schoop et al. 1999 for an excellent current review of such culture methodologies). The inclusion of viable connective tissue cells in the support matrix generally seems necessary for the reliable formation of a basal lamina by the cultured keratinocytes (Fleischmajer et al. 1998; Shoop et al. 1999). However, because fibroblasts or fibroblast-like cells included in the matrix synthesize and catabolize hyaluronan, use of a support matrix with included fibroblasts to facilitate reformation of an epithelial basal lamina would have confounded our objective of studying hyaluronan metabolism within the epidermal compartment.

Since cultures lacking a basal lamina permitted diffusion of newly synthesized hyaluronan away from the reconstituted epidermis, and inclusion of supporting cells in the collagen matrix necessary for basal lamina formation would have partially masked hyaluronan synthesis by the keratinocytes, we employed the concept of culturing keratinocytes on a preformed basal lamina, a strategy previously employed by one of us (D.K.M.) in culturing oral keratinocytes (Lillie et al. 1988). In those previous studies, a basement membrane deposited on a supporting matrix of collagen fibrils by bovine corneal endothelial cells was employed (the in vitro equivalent of Descemet's membrane). However, the membrane deposited by the bovine corneal endothelial cells exhibited numerous pores caused by corneal endothelial processes that remained in place as the membrane was deposited. Since the porous nature of the bovine corneal endothelialdeposited membrane ruled out its use, we modified the experiments of Valentich (1982), who found that a basal lamina was deposited by MDCK cells when they were cultured on a supporting matrix of collagen fibrils. MDCK cells were subsequently shown to synthesize normal components of an epithelial basal lamina: type IV collagen, laminin, and heparan sulfate proteoglycan (perlecan) (Taub 1991; Patrone et al. 1992).

In our experiments, detergent lysis of the MDCK cells followed by scanning electron microscopic examination showed an essentially intact basal laminar surface interrupted by an occasional pore, a result of MDCK cell processes extending through the membrane as previously reported by Valentich (1982). These pores were occupied by cell processes of the cultured keratinocytes (not shown) in a manner identical to that described for cultured oral keratinocytes (Lillie et al. 1988) thus making the basal lamina an effective barrier to the diffusion of macromolecular hyaluronan out of the epithelium.

Culture of the REKs on a basal lamina-covered collagen fibrillar matrix markedly altered the manner in which hyaluronan was treated by the keratinocytes when compared to those cultured on a collagen support matrix without a basal lamina. In cultures lacking a basal lamina, abundant, large hyaluronan molecules were found in the supporting collagenous matrix and underlying medi- um. In contrast, hyaluronan recovered from the basal lamina-covered matrix and underlying medium was reduced in amount and of smaller molecular mass suggesting that macromolecular hyaluronan was retained within the epithelium. Key observations from these experiments were that: (1) the total ${ }^{3} \mathrm{H}$-labeled hyaluronan accumulated in cultures without a basal lamina was greater than in cultures with a basal lamina and (2) conversely, the total amount retained in the epithelium was greater in cultures with a basal lamina. The first of these observations indicates that at least $35 \%$ of the hyaluronan synthesized during the labeling period, the difference between the two culture configurations at the end of the labeling period, was catabolized within the epithelium in cultures with a basal lamina. The actual amount is likely to be more because cultures without a basal lamina also catabolize some hyaluronan even though most diffuses out of the epithelium. We expect, then, that the half-life of a newly synthesized hyaluronan molecule in cultures with a basal lamina will be close to that observed for explants of human skin, approximately 1 day (Tammi et al. 1991). The second observation indicates that the retention of hyaluronan within the epithelial layer when a basal lamina is present increases the steady state concentration of hyaluronan around the cells in the basal and spinous cell layers. Besides demonstrating the effectiveness of the basal lamina in preventing the movement of macromolecules out of the epidermal compartment, experiments with the two different support matrices emphasize that not all hyaluronan synthesized by the keratinocytes is tethered to the cell surface and that some is free to move within the intercellular space. While the present experiment was not designed to study hyaluronan internalization, the results obtained emphasize the importance of high concentrations of hyaluronan proximate to the keratinocyte cell surface for efficient catabolism.

Our previous work with these REKs indicated that hyaluronan was tethered to the cell surface either by the enzymes responsible for its synthesis, hyaluronan synthases, or by the hyaluronan receptor CD44 (Tammi et al. 1998). Although the catabolic processes responsible for hyaluronan breakdown are not fully understood, there is evidence that the hyaluronan receptor is important in the binding and subsequent internalization of hyaluronan (Hua et al. 1993; Kaya et al. 1997).

Hyaluronan was the major glycosaminoglycan synthesized by the cultured REKs followed by heparan sulfate and chondroitin sulfate. The relative proportions of these glycosaminoglycans formed by the cultured keratinocytes are very similar to that formed by the epidermis in organ cultures of porcine (King 1981) and human skin (Tammi and Tammi 1986). This finding indicates the REKs may be an excellent model in which to study overall glycosaminoglycan/proteoglycan metabolism in the epidermis.

Although the functional roles of hyaluronan are not completely understood, it is now evident that it is not simply a large, passive, space-filling connective tissue molecule. Hyaluronan has been shown to be an impor- 
tant cell surface component which, through interactions with other molecules, plays significant roles in morphogenesis, wound healing, immune function, and malignant behavior (Sherman et al. 1994; Entwistle et al. 1996; Knudson 1998; Zeng et al. 1998). The homeostatic roles of hyaluronan in epithelia are not understood. Certain secretory epithelia add hyaluronan to their secretions (Mani et al. 1992; see Tammi et al. 1994b; Yung et al. 1996; Usui et al. 1999). But such secretory epithelia together with most simple epithelia lack demonstrable intercellular hyaluronan. That finding contrasts with the universal presence of intercellular hyaluronan within the basal cell layer and proximate suprabasal layers of all stratified epithelia studied thus far (Tammi et al. 1988, 1990; Laurent et al. 1995; Wang et al. 1996; Hirvikoski et al. 1999). It is possible that the large hydrated domain produced by intercellular hyaluronan in these stratified epithelia facilitates diffusion of metabolites in and out of these avascular tissues as well as providing a hydrated pathway for cells of the immune system that traffic through these tissues. A further role for hyaluronan within these stratified epithelia may be one of regulating cell division. Transgenic mice expressing an antisense CD44 hyaluronan receptor also lacked demonstrable intercellular epidermal hyaluronan. These same mice did not exhibit a normal proliferative response following wounding or challenge with phorbol ester; observations which seem to implicate that hyaluronan is necessary for a normal proliferative response (Kaya et al. 1997). Hyaluronan may also, in some way, play a role in regulating the differentiation of keratinocytes as they move toward the surface of the tissue. This idea comes from the observation that dysplastic stratified epithelia that contain increased layers of immature basal-like and suprabasal cells exhibit intercellular hyaluronan throughout a much greater depth of the tissue than that seen in normal stratified squamous epithelium (Wang et al. 1996; Hirvikoski et al. 1999). Some experiments also indicate hyaluronan or its CD44 receptor may play a role in keratinocyte cell to cell adhesion but the mechanisms are not understood (Milstone et al. 1994; Hudson et al. 1995).

REKs cultured on collagen or basal lamina-covered collagen matrices grow, stratify, and differentiate in a manner similar to the native epidermal tissue. The distribution of hyaluronan and the hyaluronan receptor CD44 within the "epidermis" formed by the cultured keratinocytes is similar to that in native epidermis as is the exceptionally active synthesis and catabolism of hyaluronan by the keratinocytes. The addition of the basal lamina to the collagen matrix permits the formation of a true epidermal compartment bounded by the cornified layer on the surface and the basal lamina subjacent to the basal cells. The basal lamina forms a true barrier to the diffusion of macromolecular hyaluronan away from the keratinocytes and results in increased catabolism of the molecule within the epithelium. Surprisingly, this study appears to be the first to demonstrate that a basal lamina acts as a barrier to a naturally occurring tissue macromolecule either in vivo or in vitro, based on a review of the literature concerned with the barrier functions of the basement membranes (Farqhuar 1991; Williams 1994). REKs cultured on a basal lamina-covered collagen gel provide a good model within which to study epidermal metabolism isolated from the influence of dermal cells or other supporting cells included in a collagen matrix.

Acknowledgements The authors wish to thank Dr. Stephen J. Weiss, the E. Gifford and Love Barnett Upjohn Professor of Internal Medicine and Oncology, University of Michigan, for freely sharing his laboratory's experience in fabricating MDCK cell basal lamina-covered collagen gels. Dr. Weiss provided protocols and the MDCK cells used in this study, as well as the initial set of basal lamina-covered collagen gels in tissue culture inserts for which we are very grateful. Drs. Raija and Markku Tammi thank the Cleveland Clinic Foundation for support during their sabbatical in the Department of Biomedical Engineering, and acknowledge additional support from the Academy of Finland, the University of Kuopio Biotechnology funds, and the Technology Development Centre of Finland (TEKES). Ms Eija Rahunen and Mr. Kari Kotikumpu are acknowledged for skillful assistance in preparing the paraffin sections and doing the histochemical reactions.

\section{References}

Ågren UM, Tammi RH, Tammi MI (1997) Reactive oxygen species contribute to epidermal hyaluronan catabolism in human skin organ culture. Free Radical Biol Med 23:996-1001

Baden HP, Kubilus J (1983) The growth and differentiation of newborn rat keratinocytes. J Invest Dermatol 80:124-130

Bignami A, Hosley M, Dahl D (1993) Hyaluronic acid and hyaluronic acid-binding proteins in brain extracellular matrix. Anat Embryol (Berl) 188:419-433

Entwistle J, Hall CL, Turley EA (1996) HA receptors: regulators of signaling to the cytoskeleton. J Cell Biochem 61:569-577

Farquhar MG (1991) The glomerular basement membrane: a selective macromolecular filter. In: Hay ED (ed) Cell biology of extracellular matrix, 2nd edn. Plenum Press, New York, pp 365-417

Fleischmajer R, Perlish JS, MacDonald ED, Schecter A, Murdoch AD, Iozzo RV, Yamada Y (1998) There is binding of collagen IV to beta 1 integrin during early skin basement membrane assembly. Ann NY Acad Sci 857:212-227

Hirvikoski P, Tammi R, Kumpulainen E, Virtaniemi J, Parkkinen JJ, Tammi M, Johansson R, Ågren U, Karhunen J, Kosma VM (1999) Irregular expression of hyaluronan and its CD44 receptor is associated with metastatic phenotype in laryngeal squamous cell carcinoma. Virchows Arch 434:37-44

Hua Q, Knudson CB, Knudson W (1993) Internalization of hyaluronan by chondrocytes occurs via receptor-mediated endocytosis. J Cell Sci 106:365-375

Huber AR, Weiss SJ (1989). Disruption of the subendothelial basement membrane during diapedesis in an in vitro construct of a blood vessel. J Clin Invest 83:1122-1136

Hudson DL, Sleeman J, Watt FM (1995) CD44 is the major peanut lectin-binding glycoprotein of human epidermal keratinocytes and plays a role in intercellular adhesion. J Cell Sci 108: 1959-1970

Kaya G, Rodriguez I, Jorcano JL, Vassalli P, Stamenkovic I (1997) Selective suppression of CD44 in keratinocytes of mice bearing an antisense CD44 transgene driven by a tissue-specific promoter disrupts hyaluronate metabolism in skin and impairs keratinocyte proliferation. Genes Dev 11:996-1007

King IA (1981) Characterization of epidermal glycosaminoglycans synthesized in organ culture. Biochim Biophys Acta 674: $87-95$

Knudson W (1998) The role of CD44 as a cell surface hyaluronan receptor during tumor cell migration and metastasis. Front Biosci 3:604-615 
Kramer RH, Fuh GM, Bensch KG, Karasek MA (1985) Synthesis of extracellular matrix of glycoproteins by cultured microvascular endothelial cells isolated from the dermis of neonatal and adult skin. J Cell Physiol 123:1-9

Laurent C, Hellström S, Engström-Laurent A, Wells AF, Bergh A (1995) Localization and quantity of hyaluronan in urogenital organs of male and female rats. Cell Tissue Res 292:241-248

Laurent TC, Laurent UB, Fraser JR (1996) The structure and function of hyaluronan: an overview. Immunol Cell Biol 72:A1A7

Lillie JH, MacCallum DK, Jepsen A (1980) Fine structure of subcultured stratified squamous epithelium grown on collagen rafts. Exp Cell Res 125:153-165

Lillie JH, MacCallum DK, Jepsen A (1988) Growth of stratified squamous epithelium on reconstituted extracellular matrices: long-term culture. J Invest Dermatol 90:100-109

MacCallum DK, Lillie JH (1990) Evidence for autoregulation of cell division and cell transit in keratinocytes grown on collagen at an air-liquid interface. Skin Pharmacol 3:86-96

Mani SK, Carson DD, Glasser SR (1992) Steroid hormones differentially modulate glycoconjugate synthesis and vectorial secretion by polarized uterine epithelial cells in vitro. Endocrinology 130:240-248

Milstone LM, Hough-Monroe L, Kugelman LC, Bender JR, Haggerty JG (1994) Epican, a heparan/chondroitin sulfate proteoglycan form of CD44, mediates cell-cell adhesion. J Cell Sci 107:3183-3190

Patrone LM, Cook JR, Crute BE, Van Buskirk RG (1992) Differentiation of epithelial cells on microporous membranes. J Tissue Culture Methods 14:225-234

Ripellino JA, Bailo M, Margolis RU, Margolis RK (1988) Light and electron microscopic studies on the localization of hyaluronic acid in developing rat cerebellum. J Cell Biol 106:845855

Schoop VM, Mirancea N, Fusenig NE (1999) Epidermal organization and differentiation of $\mathrm{HaCaT}$ keratinocytes in organotypic coculture with human dermal fibroblasts. J Invest Dermatol 112:343-353

Sherman L, Sleeman J, Herrlich P, Ponta H (1994) Hyaluronate receptors: key players in growth, differentiation, migration and tumor progression. Curr Opin Cell Biol 6:726-733

Tammi R, Tammi M (1986) The influence of retinoic acid on the ultrastructure and hyaluronic acid synthesis of adult human epidermis in whole skin organ cultures. J Cell Physiol 126: 389-398

Tammi R, Ripellino JA, Margolis RU, Tammi M (1988) Localization of epidermal hyaluronic acid using the hyaluronate binding region of cartilage proteoglycan as a specific probe. J Invest Dermatol 90:412-414

Tammi R, Ripellino JA, Margolis RU, Maibach HI, Tammi M (1989) Hyaluronate accumulation in human epidermis treated with retinoic acid in skin organ culture. J Invest Dermatol 92:326-332

Tammi R, Tammi M, Häkkinen L, Larjava H (1990) Histochemical localization of hyaluronate in human oral epithelium using a specific hyaluronate-binding probe. Arch Oral Biol 35:219224

Tammi R, Säämänen A-M, Maibach HI, Tammi M (1991) Degradation of newly synthesized high molecular mass hyaluronan in the epidermal and dermal compartments of human skin in organ culture. J Invest Dermatol 97:126-130

Tammi R, Rönkkö S, Ågren UM, Tammi M (1994a) Distribution of hyaluronan in bull reproductive organs. J Histochem Cytochem 42:1479-1486

Tammi R, Åren UM, Tuhkanen A-L, Tammi M (1994b) Hyaluronan metabolism in skin. In: Graumann W (ed) Progress in histochemistry and cytochemistry. Fischer, Stuttgart, 29:1-81

Tammi R, MacCallum D, Hascall VC, Pienimäki JP, Hyttinen M, Tammi M (1998) Hyaluronan bound to CD44 on keratinocytes is displaced by hyaluronan decasaccharides but not hexasaccharides. J Biol Chem 273:28878-28888

Tammi R, Tammi M, Hascall VC, Hunziker EB, MacCallum DK (1999) Does keratinocyte hyaluronan determine the volume of the extracellular space in the epidermis? In: Abatangelo $\mathrm{G}$ (ed) New frontiers in medical science: redefining hyaluronan. Elservier Science Publishers, Amsterdam, (in press)

Taub M (1991) Retinoic acid inhibits basement membrane protein biosynthesis while stimulating dome formation by Madin Darby canine kidney cells in hormonally defined serum-free medium. J Cell Physiol 148:211-219

Toole BP (1991) Proteoglycans and hyaluronan in morphogenesis and differentiation. In: Hay ED (ed) Cell biology of extracellular matrix, 2nd edn. Plenum Press, New York, pp 305-334

Usui T, Suzuki K, Kaji Y, Amano S, Miyata K, Heldin P, Yamashita H (1999) Hyaluronan synthase expression in bovine eyes. Invest Ophthalmol Vis Sci 40:563-567

Valentich JD (1982) Basal lamina assembly by the dog kidney cell line MDCK. Cold Spring Harbor Conf Cell Prolif 9:567-579

Voigt W-H, Fusenig NE (1979) Organotypic differentiation of mouse keratinocytes in cell culture: a light- and electronmicroscopic study. Biol Cell 34:111-118

Wang C, Tammi M, Guo H, Tammi R (1996) Hyaluronan distribution in the normal epithelium of the esophagus, stomach, and colon and their cancers. Am J Pathol 148:1861-1869

Wight TN, Heinegård DK, Hascall VC (1991) Proteoglycans, structure and function. In: Hay ED (ed) Cell biology of extracellular matrix, 2nd edn. Plenum Press, New York, pp 45-78

Williams JC (1994) Permeability of basement membranes to macromolecules. Proc Soc Exp Biol Med 207:13-19

Yanagishita M, Salustri A, Hascall VC (1989) Determination of the specific activity of hexosamine precursors by analysis of double labeled disaccharides from chondroitinase digestion of chondroitin/dermatan sulfate. Methods Enzymol 179:435-455

Yung S, Coles GA, Davies M (1996) IL-1 beta, a major stimulator of hyaluronan synthesis in vitro of human peritoneal mesothelial cells: relevance to peritonitis in CAPD. Kidney Int 50: $1337-1343$

Zeng C, Toole BP, Kinney SD, Kuo JW, Stamenkovic I (1998) Inhibition of tumor growth in vivo by hyaluronan oligomers. Int J Cancer 77:396-401 